\title{
Tyrphostin AG 1296 induces glioblastoma cell apoptosis in vitro and in vivo
}

\author{
HONGWEI LI ${ }^{1,2}$, JUNNING ZHENG $^{2}$, RUIYUN GUAN ${ }^{2}$, ZIFENG ZHU $^{2}$ and XIANHOU YUAN ${ }^{1}$ \\ ${ }^{1}$ Department of Neurosurgery, Zhongnan Hospital, Wuhan University, Wuhan, Hubei 430071; \\ ${ }^{2}$ Department of Neurosurgery, The Eight People's Hospital of Shenzhen, Shenzhen, Guangdong 510000, P.R. China
}

Received August 28, 2014; Accepted May 14, 2015

DOI: $10.3892 / 01.2015 .3781$

\begin{abstract}
Glioblastoma is the most common type of malignant human brain tumor. Currently available chemotherapies for glioblastoma focus on targeting tyrosine kinases. However, the existing inhibitors of tyrosine kinases have not produced the therapeutic outcomes that were anticipated. In order to investigate the viability alternative chemotherapeutic agents in this disease, the present study examined the anticancer effects of tyrphostin AG 1296, focusing on its involvement in apoptosis in glioblastoma cells. The study aimed to identify whether tyrphostin AG 1296 affects glioblastoma cell growth by inducing cell apoptosis. To achieve this, cell viability, propidium iodide analysis and cell invasion assay were used to measure cell growth, cell apoptosis and cell migration of human glioblastoma cells. The results showed that tyrphostin AG 1296 treatment reduced cell viability and suppressed migration of human glioblastoma cells. It was also demonstrated that tyrphostin AG 1296 induced cell apoptosis in vitro. Finally, tyrphostin AG 1296 was also shown to significantly inhibit the growth of glioblastoma cells and to increase tumor cell apoptosis in vivo. These findings suggest that tyrphostin AG 1296 induces apoptosis, thereby reducing cell viability and capacity for migration of glioblastoma cells.
\end{abstract}

\section{Introduction}

Glioblastoma multiforme (GBM) is the most prevalent type of primary human brain tumor, and has low rates of survival (1-3). Current therapeutic strategies for GBM, including radiotherapy and chemotherapy, do not produce satisfactory clinical outcomes $(4,5)$. Therefore, a novel and effective approach with which to treat GBM is required.

Correspondence to: Dr Xianhou Yuan, Department of Neurosurgery, Zhongnan Hospital, Wuhan University, 169 Donghu Road, Wuhan, Hubei 430071, P.R. China

E-mail: xianhou.yuan@outlook.com

Key words: tyrphostin AG 1296, platelet-derived growth factor receptor inhibitor, glioblastoma, cancer therapy
Mechanistic studies suggest that numerous genetic and physiological alterations occur in GBM, which affect the survival and proliferation of glioblastoma cells (6). Receptor tyrosine kinases (RTKs) are genes/proteins that are frequently modified during the pathogenesis of GBM (7). Evidence from a number of studies, supports the hypothesis that platelet-derived growth factor receptor (PDGFR), a member of the type III RTK family, and the signaling pathway through PDGFR is strongly associated with the development of GBM (8-10). PDGF and its receptors have been shown to be upregulated in one third of surgical glioblastoma samples and human glioblastoma cell lines (8). In addition, in vitro and in vivo studies have demonstrated that PDGF and its receptors are involved in the proliferation, cell migration and angiogenesis of GBM cells $(11,12)$. Furthermore, PDGF autocrine loops have been reported to be involved in the tumorigenesis of GBM $(13,14)$. In light of these findings, it was hypothesized that inhibition of PDGF signaling may be a viable therapeutic approach for GBM.

Currently, different types of PDGF antagonists are being developed and evaluated in animal disease models, in addition to clinical trials (15). Tyrphostin AG 1296 is a specific inhibitor of human PDGFR- $\alpha$ and $-\beta$, as well as of c-KIT (also termed sCD117), fms-related tyrosine kinase 3 and Bek (a fibroblast growth factor receptor) tyrosine kinases (16-19). It has been reported that tyrphostin AG 1296 inhibits cell proliferation and promotes apoptosis in small cell lung cancer, leukemia and anaplastic thyroid cancer (20-22). Recently, it has been suggested that AG 1296 may suppress the proliferation and migration of glioblastoma cells $(23,24)$. Whilst tyrphostin AG 1296 has been shown to affect the proliferation and migration of glioblastoma cells, its effect on the rate of apoptosis in GBM remains unclear.

In the present study, the effects of tyrphostin AG 1296 on cell apoptosis in glioblastoma cells were examined in vitro and in vivo. The in vitro experiments demonstrated that tyrphostin AG 1296 reduced cell viability, inhibited cell migration and promoted cell apoptosis. The in vivo experiments also showed that tyrphostin AG 1296 inhibited tumor growth and induced tumor cell apoptosis in a mouse xenograft model of human GBM. Therefore the current data support the hypothesis that tyrphostin AG 1296 inhibits the growth of glioblastoma cells by inducing apoptosis. 
A

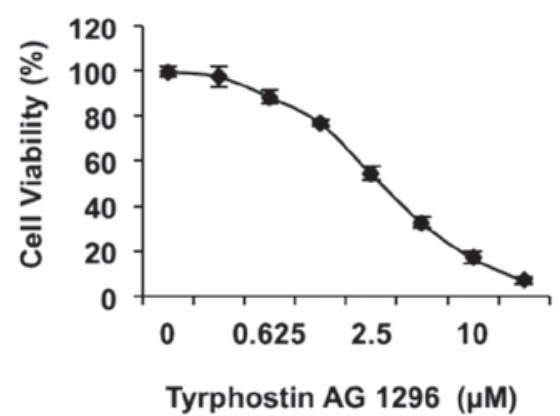

B

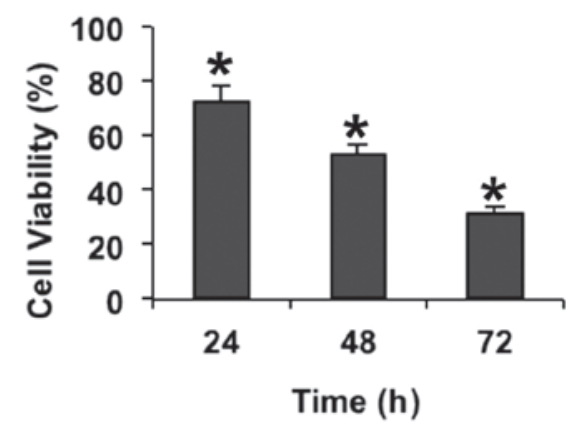

Figure 1. Tyrphostin AG 1296 decreased cell viability of U87MG cells. (A) Cell viability was measured following treatment of U87MG cells with tyrphostin AG $1296(0.3125-20 \mu \mathrm{M})$ for $72 \mathrm{~h}$. (B) Cell viability was measured following treatment of U87MG cells with tyrphostin AG 1296 (5 $\mu$ M) for 24,48 or $72 \mathrm{~h}$. The viability of cells was set to $100 \%$ in the untreated control group. $\mathrm{P}<0.05$ vs. control.

\section{Materials and methods}

U87MG cell culture. U87MG, a representative grade IV glioma cell line, was obtained from the American Type Culture Collection (Manassas, VA, USA). U87MG cells were cultured in Dulbecco's modified Eagle's medium/F12/10\% FBS medium (Invitrogen, Grand Island, NY, USA). Cells were maintained in a humidified incubator at $37^{\circ} \mathrm{C}$ with $5 \% \mathrm{CO}_{2}$.

Cell viability assay. U87MG Cells were treated with 0.3125 , $0.625,1.25,2.5,5,10$ or $20 \mu \mathrm{M}$ of tyrphostin AG 1296 (ThermoFisher Scientific, Inc., Waltham, MA USA; Catalog No. 50-230-7882), $72 \mathrm{~h}$ after cells had been seeded in 96-well plates for a period of $24 \mathrm{~h}$. In order to assess whether the duration of treatment affected cell viability, cells were treated with $5 \mathrm{mM}$ tyrphostin AG 1296 for 24, 48 or $72 \mathrm{~h}$. The cell viability assay was performed using a CellTiter-Glo ${ }^{\circledR}$ Assay kit (Promega Corporation, Madison, WI USA). Experiments were repeated three times, independently.

Propidium iodide analysis. Cells were treated with tyrphostin AG 1296 at various concentrations and harvested after $48 \mathrm{~h}$. Propidium iodide staining was performed using a Cycletest ${ }^{\mathrm{TM}}$ plus DNA reagent kit (Becton Dickinson, San Jose, CA, USA), followed by detection of the apoptotic cells with DNA content in sub-G1, using a FACSCalibur flow cytometry. Data were analyzed using CellQuest Pro software, version 5.1 (Becton Dickinson).

Morphological analysis of U87MG cells. U87MG cells treated with tyrphostin AG 1296 at various concentrations, were stained using Hoechst. Cells with condensed or fragmented nuclei were identified as apoptotic cells using a Olympus BX53 microscope (Olympus Corporation, Tokyo, Japan).

Cell invasion assay. For the cell invasion assay, $1 \times 10^{6}$ cells were plated into the upper chambers of 24-transwell Boyden chamber wells (Costar, Bedford, MA, USA), and treated with tyrphostin AG 1296 at various concentrations for $8 \mathrm{hr}$. Following treatment, cells were fixed and stained with $0.1 \%$ crystal violet for imaging. Migrated cells were lysed with $10 \%$ acetic acid following imaging. The absorbance of lysate was measured at $595 \mathrm{~nm}$ by using a Nanodrop 2000 (Thermo Scientific, Inc., Wilmongton, DE, USA).

Tyrphostin AG 1296 treatment in a mouse xenograft model in vivo. Approval for the use of mice in the present study, was obtained from the Animal Ethics Committee and the Institutional Review Board of Wuhan University (Wuhan, China). C57BL/6J mice were purchased from Shanghai SLAC laboratory Animal Co., Ltd. (Shanhai, China). Animals were provided free access to food and water and were housed in a 13:11 (light:dark) cycle. Female mice aged 6-8 weeks, weighing $20 \mathrm{~g}$ were used for experiments. U87MG cells ( $2 \times 10^{6}$ cells/mouse) were injected into the axillary regions of mice. When tumor volumes had reached $70 \mathrm{~mm}^{3}$, the mice were randomly divided into a control group and low and high dose tyrphostin AG 1296 treatment groups ( $\mathrm{n}=6 /$ group$)$. Low-dose $(50 \mathrm{mg} / \mathrm{kg})$ or high-dose $(100 \mathrm{mg} / \mathrm{kg})$ tyrphostin AG 1296, or vehicle (10\% 1-methyl-2-pyrrolidinone and $90 \%$ polyethylene glycol 300) was intravenously injected into the mice each day for 13 days. Tumor size and body weight were measured daily. The tumor volume was calculated according to the formula: Volume $\left(\mathrm{mm}^{3}\right)=$ (width $\mathrm{x}$ width $\mathrm{x}$ length $) / 2$.

Terminal deoxynucleotidyl transferase-mediated dUTP nick end-labeling (TUNEL) assay. Tumor samples from the control and the AG 1296-treatment groups were harvested from mice following 13 days of treatment. The tumors were cut into $5-\mathrm{mm}^{3}$ sections and fixed in $4 \%$ paraformaldehyde. Subsequently, samples were embedded in paraffin blocks and 5-mm sections were produced. Apoptotic cells were identified using a TUNEL assay. Briefly, tumor tissues were incubated with TdT enzyme solution (New England Biolabs, Inc., Ipswich, MA, USA) for $60 \mathrm{~min}$, washed twice with PBS twice and then incubated for $30 \mathrm{~min}$ at $25^{\circ} \mathrm{C}$ with DAPI. Images were captured using an Olympus BX53 fluorescence microscope.

Statistical analysis. All experiments were repeated in triplicates, independently. The data were analyzed by a one-way or two-wayanalysis of variance followed by Tukey's post-hoc test. Graphs were produced using SPSS statistical software, version 17.0 (SPSS, Chicago, IL, USA). Data are expressed as the mean \pm standard deviation. $\mathrm{P}<0.05$ was considered to indicate a statistically significant difference. 
A
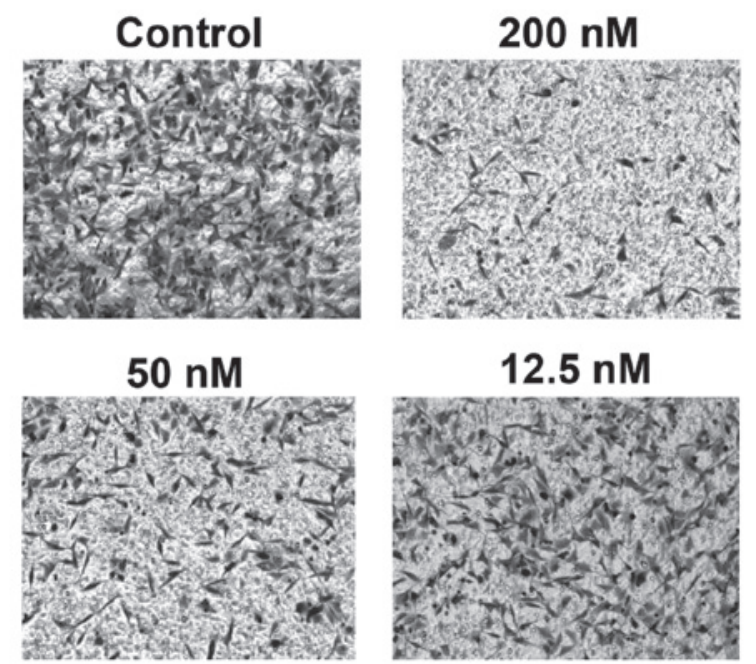

$12.5 \mathrm{nM}$

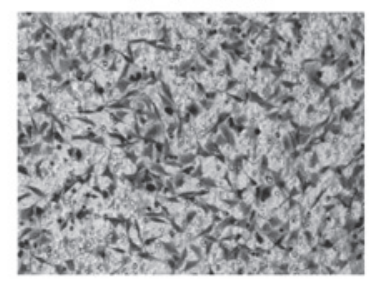

B

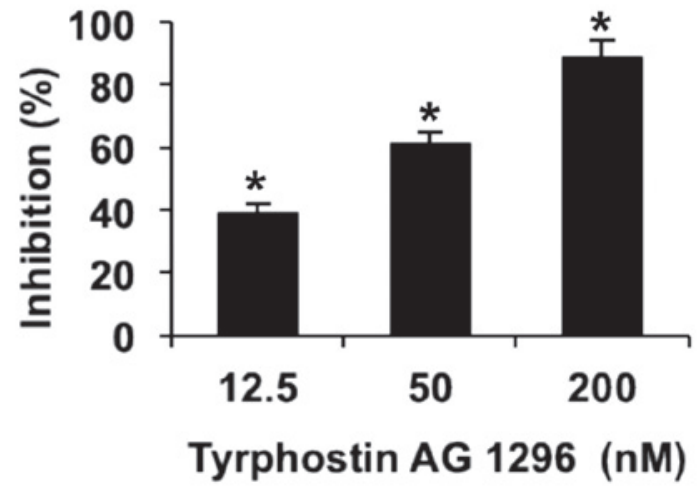

Figure 2. Tyrphostin AG 1296 inhibited cell migration of glioblastoma cells. (A) Migrated cells on the lower side were stained and photographed following treatment of U87MG cells with tyrphostin AG $1296(12.5,50$ or $200 \mathrm{nM})$ for $8 \mathrm{~h}$. Representative images are shown. The dark cells are migrated cells stained with crystal violet. Less dark cells are present in the tyrphostin AG-treated groups compared with the control group. (B) Quantitative analysis of inhibition of cell invasion, as assessed using a transwell assay. Inhibition was set to $100 \%$ for the untreated control group. "P<0.05 vs. control.

A

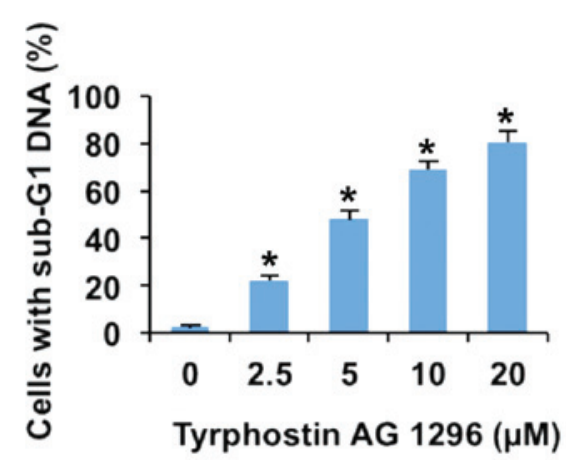

B

Control Tyrphostin AG $1296(20 \mu \mathrm{M})$

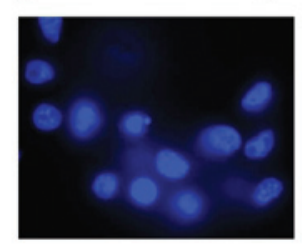

C

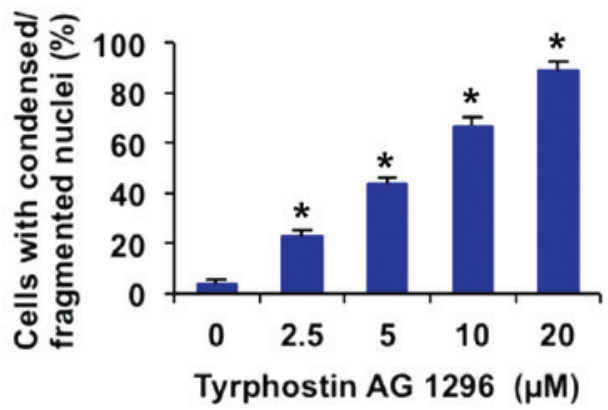

Figure 3. Tyrphostin AG 1296 promoted cell apoptosis in U87MG cells. (A) Flow cytometry analysis of apoptotic cells following treatment of U87MG cells with tyrphostin AG $1296(0,2.5,5,10$ or $20 \mu \mathrm{M})$ for $48 \mathrm{~h}$. (B) U87MG cells were incubated with tyrphostin AG 1296 for $48 \mathrm{~h}$ and then stained with Hoechst. Images were captures using a fluorescence microscope and representative images are shown. (C) Quantitative analysis of the number of cells with condensed/fragmented nuclei observed in seven randomly-selected fields. Cell inhibition was set to $100 \%$ in the untreated control group.*P<0.05 vs. control.

\section{Results}

Tyrphostin AG 1296 inhibits U87MG cell viability. Previous studies have suggested that tyrphostin AG 1296, an inhibitor of PDGFR, reduces glioblastoma cell proliferation (24). In order to confirm whether tyrphostin AG 1296 affects glioblastoma cell growth, a cell viability assay was performed in U87MG cells. The results showed that tyrphostin AG 1296 inhibited the viability of U87MG cells, in a dose-dependent manner (Fig. 1A). It was observed that cell viability was almost fully suppressed when cells were treated with $20 \mu \mathrm{M}$ tyrphostin AG 1296 for $72 \mathrm{~h}$ (Fig. 1A). In order to investigate whether the effect of tyrphostin AG 1296 on the viability of U87MG cells is time-dependent, cells were treated with $5 \mu \mathrm{M}$ tyrphostin AG 1296 for 24, 48 and $72 \mathrm{~h}$. It was shown tyrphostin AG 1296 reduced U87MG cell viability in a time-dependent manner (Fig. 1B). Therefore, the current data confirm that tyrphostin AG 1296 inhibits glioblastoma cell viability in vitro. 
A

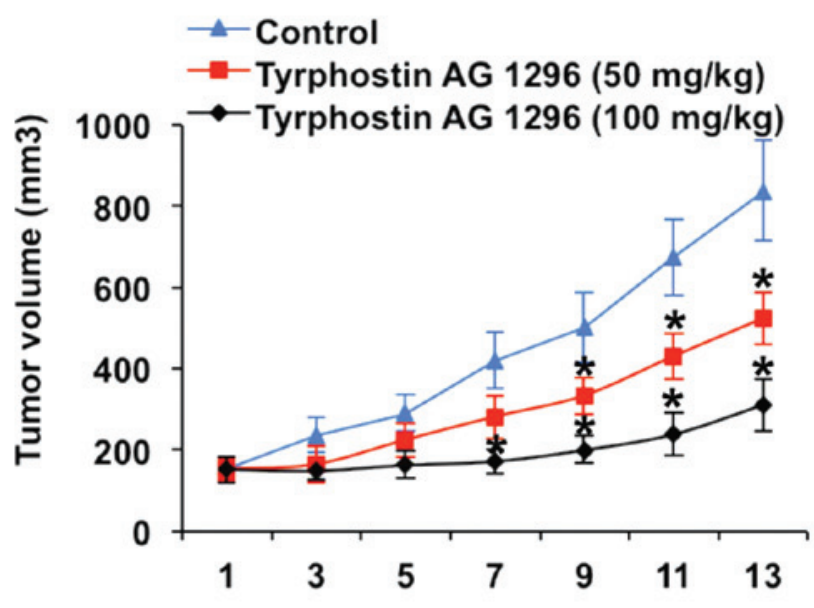

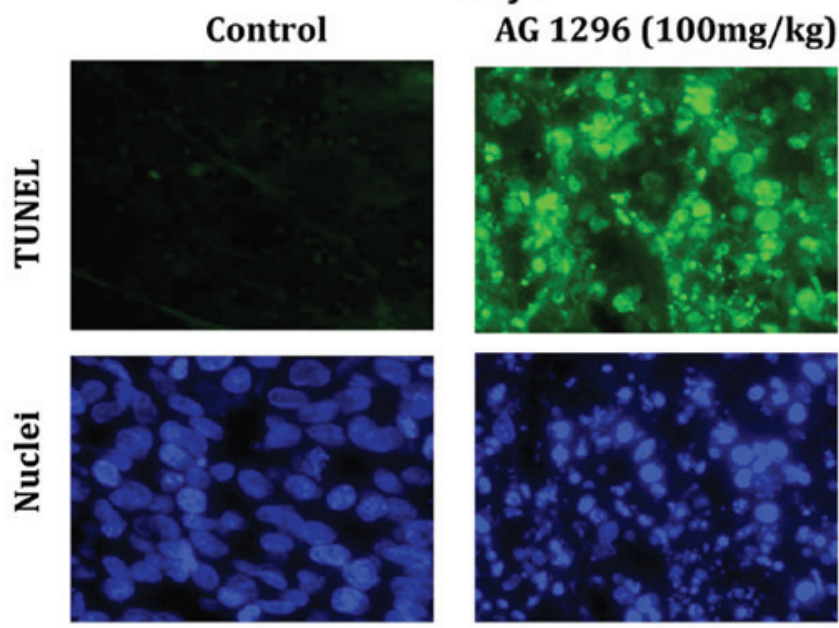

Figure 4. Tyrphostin AG 1296 repressed glioblastoma tumor growth in vivo. (A) Tyrphostin AG 1296 (50 and 100 mg/kg) was injected into mice daily, for a period of 13 days following inoculation of U87MG cells. Tumor volumes were measured on alternate days and the growth curves of tumors are shown. ${ }^{*} \mathrm{P}<0.05$ vs. control. (B) The effect of tyrphostin AG 1296 on U87MG cell apoptosis in vivo was measured using a TUNEL assay (green). Nuclei were stained with Hoechst (blue). TUNEL, terminal deoxynucleotidyl transferase-mediated dUTP nick end-labeling.

Tyrphostin AG 1296 inhibits U87MG cell invasion in vitro. Subsequently, the effects of tyrphostin AG 1296 on U87MG cell invasion were examined. Matrigel invasion assays, with $8.0 \mu \mathrm{m}$-pore transwells were used. The results of the invasion assays showed that low and high concentrations of tyrphostin AG 1296 effectively restricted U87MG cell migration across the filter (Fig. 2A). Statistical analysis demonstrated that the anti-migration effect of tyrphostin AG 1296 in U87MG cells, increased in a dose-dependent manner (Fig. 2B). These results are in accordance with those of a previous study, which suggested that tyrphostin AG 1296 inhibits glioblastoma cell invasion in vitro (23).

Tyrphostin AG 1296 induces apoptosis of U87MG cells. Although the effects of tyrphostin AG 1296 on cell growth and migration have been investigated, its effect on glioblastoma cell apoptosis has not been fully elucidated. In order to investigate whether tyrphostin AG 1296 regulates cell apoptosis, the fraction of apoptotic cells with sub-G1 DNA content, with and without tyrphostin AG 1296 treatment, was examined. The results showed that the percentage of cells with sub-G1 DNA content increased in a dose-dependent manner (Fig. 3A). Furthermore, U87MG cells exposed to tyrphostin AG 1296 exhibited the condensed and fragmented morphology (Fig. 3B), which is characteristic of apoptotic cells. The number of cells with condensed or fragmented nuclei was markedly increased in U87MG cells, and this increase occurred in a dose-dependent manner (Fig. 3C). These results demonstrated that tyrphostin AG 1296 induces apoptosis in U87MG cells.

Tyrphostin AG 1296 antagonizes tumor growth and induces apoptosis of tumor cells in vivo. In order to measure the antitumor activity of tyrphostin AG 1296 in vivo, a glioblastoma xenograft model was generated by injecting mice with U87MG cells. Once tumor volumes reached $70 \mathrm{~mm}^{3}$, various doses of tyrphostin AG 1296 were administered over 13 days. Compared with the control group, tyrphostin AG 1296 led to reduction of tumor growth at low $(50 \mathrm{mg} / \mathrm{kg})$ and high $(100 \mathrm{mg} / \mathrm{kg})$ doses. Furthermore, high-dose tyrphostin AG 1296 suppressed tumor growth to a greater extent than low-dose treatment (Fig. 4A). In accordance with the in vitro data, cell apoptosis was observed in tumor tissues (Fig. 4B). Tyrphostin AG 1296 induced changes characteristic of apoptosis, as measured using a TUNEL assay (Fig. 4B). In addition, mice exhibited no significant signs of toxicity or weight loss during Tyrphostin AG 1296 treatment (data not shown). These data indicate that tyrphostin AG 1296 suppresses GBM tumor growth by inducing cell apoptosis in vivo.

\section{Discussion}

GBM is the most common type of primary tumor of the central nervous system (25-27). The overall median survival period is $\sim 15$ months for individuals with GBM (28). The majority of current chemotherapeutic approaches for GBM involve targeting tyrosine kinase receptor signaling pathways, due to the central role of these molecules in the oncogenesis of GBM (29). However, RTK inhibitors have not produced the level of efficacy in patients with GBM, which had been anticipated (30,31). Thus, the development of novel chemotherapeutic agents for GBM is required. The present study examined the effect of tyrphostin AG 1296 on glioblastoma cell growth and migration in vitro and in vivo. In accordance with previous studies $(23,24)$, tyrphostin AG 1296 exhibited marked efficacy in the inhibition of glioblastoma cell growth and migration in vitro. Furthermore, it was demonstrated that tyrphostin AG 1296 induced cell apoptosis in vitro and in vivo. The inhibitory effects of tyrphostin AG 1296 on proliferation and migration, and the induction of apoptosis by this agent, resulted in a reduction in the growth of GBM tumors in vivo (Fig. 4). The results of previous studies and of the present study, on tyrphostin AG 1296 improve the current understanding of GBM treatment and suggest a novel therapeutic approach to this disease. 
In addition to tyrphostin AG 1296, other inhibitors targeting PDGFR- $\beta$, include Imatinib. Imatinib is currently used for the clinical treatment of certain types of cancer, including chronic myeloid leukemia and gastrointestinal stromal tumor (32-34). However, Imatinib has exhibited limited efficacy in patients with GBM in phase I/II clinical trials (35), which may be a result of resistance, due to mutations in the GBM tumors $(36,37)$. The present study demonstrated a strong antitumor activity of tyrphostin AG 1296 in a glioblastoma cell line and a GBM mouse model. Therefore, tyrphostin AG 1296 may be a promising chemotherapeutic option for malignant GBM. However, its efficacy in patients with GBM requires further evaluation.

\section{Acknowledgements}

The authors would like to thank Professor Xiwen Shi (Henan Hospital, Zhengzhou, China), Professor Lijun Huang (Xinxiang Hospital, Xinxiang, China) and Professor Cheng Wang (Shenzhen hospital, Shenzen, China) for their advice, as well as all members of the laboratory for their technical support and suggestions.

\section{References}

1. Davis FG, Freels S, Grutsch J, et al: Survival rates in patients with primary malignant brain tumors stratified by patient age and tumor histological type: An analysis based on surveillance, epidemiology and end results (SEER) data, 1973-1991. J Neurosurg 88: 1-10, 1998

2. Maher EA, Furnari FB, Bachoo RM, et al: Malignant glioma: Genetics and biology of a grave matter. Genes Dev 15: 1311-1333, 2001.

3. Ricard D, Idbaih A, Ducray F, et al: Primary brain tumours in adults. Lancet 379: 1984-1996, 2012.

4. Aherne NJ, Benjamin LC, Horsley PJ, et al: Improved outcomes with intensity modulated radiation therapy combined with temozolomide for newly diagnosed glioblastoma multiforme. Neurol Res Int 2014: 945620, 2014

5. Aizer AA, Ancukiewicz M, Nguyen PL, et al: Underutilization of radiation therapy in patients with glioblastoma: Predictive factors and outcomes. Cancer 120: 238-243, 2014.

6. Kanu OO, Hughes B, Di C, et al: Glioblastoma Multiforme Oncogenomics and Signaling Pathways. Clin Med Oncol 3 39-52, 2009.

7. Krause DS and Van Etten RA: Tyrosine kinases as targets for cancer therapy. N Engl J Med 353: 172-187, 2005.

8. Guha A, Dashner K, Black PM, Wagner JA and Stiles CD: Expression of PDGF and PDGF receptors in human astrocytoma operation specimens supports the existence of an autocrine loop. Int J Cancer 60: 168-173, 1995.

9. Lokker NA, Sullivan CM, Hollenbach SJ, et al: Platelet-derived growth factor (PDGF) autocrine signaling regulates survival and mitogenic pathways in glioblastoma cells: Evidence that the novel PDGF-C and PDGF-D ligands may play a role in the development of brain tumors. Cancer Res 62: 3729-3735, 2002.

10. Nazarenko I, Hede SM, He X, et al: PDGF and PDGF receptors in glioma. Ups J Med Sci 117: 99-112, 2012.

11. Capdeville R, Buchdunger E, Zimmermann J and Matter A: Glivec (STI571, imatinib), a rationally developed, targeted anticancer drug. Nat Rev Drug Discov 1: 493-502, 2002.

12. Guo P, Hu B, Gu W, et al: Platelet-derived growth factor-B enhances glioma angiogenesis by stimulating vascular endothelial growth factor expression in tumor endothelia and by promoting pericyte recruitment. Am J Pathol 162: 1083-1093, 2003.

13. Heldin $\mathrm{CH}$ and Westermark B: Mechanism of action and in vivo role of platelet-derived growth factor. Physiol Rev 79: 1283-1316, 1999.

14. Nistér M, Claesson-Welsh L, Eriksson A, Heldin $\mathrm{CH}$ and Westermark B: Differential expression of platelet-derived growth factor receptors in human malignant glioma cell lines. J Biol Chem 266: 16755-16763, 1991.
15. Ostman A and Heldin $\mathrm{CH}$ : Involvement of platelet-derived growth factor in disease: Development of specific antagonists. Adv Cancer Res 80: 1-38, 2001.

16. Kovalenko M, Rönnstrand L, Heldin CH, et al: Phosphorylation site-specific inhibition of platelet-derived growth factor beta-receptor autophosphorylation by the receptor blocking tyrphostin AG1296. Biochemistry 36: 6260-6269, 1997.

17. Kovalenko M, Gazit A, Böhmer A, et al: Selective platelet-derived growth factor receptor kinase blockers reverse sis-transformation. Cancer Res 54: 6106-6114, 1994.

18. Strutz F, Zeisberg M, Renziehausen A, Raschke B, Becker V, van Kooten $C$ and Müller G: TGF-beta 1 induces proliferation in human renal fibroblasts via induction of basic fibroblast growth factor (FGF-2). Kidney Int 59: 579-592, 2001.

19. Levitzki A: Protein tyrosine kinase inhibitors as novel therapeutic agents. Pharmacol Ther 82: 231-239, 1999.

20. Krystal GW, Carlson P and Litz J: Induction of apoptosis and inhibition of small cell lung cancer growth by the quinoxaline tyrphostins. Cancer Res 57: 2203-2208, 1997.

21. Minami Y, Yamamoto K, Kiyoi H, Ueda R, Saito H and Naoe T: Different antiapoptotic pathways between wild-type and mutated FLT3: Insights into therapeutic targets in leukemia. Blood 102: 2969-2975, 2003.

22. Che HY, Guo HY, Si XW, You QY and Lou WY: Additive effect by combination of Akt inhibitor, MK-2206 and PDGFR inhibitor, tyrphostin AG 1296, in suppressing anaplastic thyroid carcinoma cell viability and motility. Onco Targets Ther 7: 425-432, 2014.

23. Sciaccaluga M, D'Alessandro G, Pagani F, et al: Functional cross talk between CXCR4 and PDGFR on glioblastoma cells is essential for migration. PLoS One 8: e73426, 2013.

24. Watanabe T, Ohtani T, Aihara M and Ishiuchi S: Enhanced antitumor effect of YM872 and AG1296 combination treatment on human glioblastoma xenograft models. J Neurosurg 118: 838-845, 2013

25. Fuller GN: The WHO classification of tumours of the central nervous system, 4th edition. Arch Pathol Lab Med 132: 906, 2008.

26. Louis DN, Holland EC and Cairncross JG: Glioma classification: A molecular reappraisal. Am J Pathol 159: 779-786, 2001.

27. Okada H, Kohanbash G, Zhu X, et al: Immunotherapeutic approaches for glioma. Crit Rev Immunol 29: 1-42, 2009.

28. Anton K, Baehring JM and Mayer T: Glioblastoma multiforme: Overview of current treatment and future perspectives. Hematol Oncol Clin North Am 26: 825-853, 2012.

29. Carrasco-García E, Saceda M and Martinez-Lacaci I: Role of receptor tyrosine kinases and their ligands in glioblastoma. Cells 3: 199-235, 2014.

30. Taylor TE, Furnari FB and Cavenee WK: Targeting EGFR for treatment of glioblastoma: Molecular basis to overcome resistance. Curr Cancer Drug Targets 12: 197-209, 2012

31. Vivanco I, Robins HI, Rohle D, Nghiemphu PL, Kubek S, Oldrini B, Chheda MG, Yannuzzi N, et al: Differential sensitivity of glioma- versus lung cancer-specific EGFR mutations to EGFR kinase inhibitors. Cancer Discov 2: 458-471, 2012.

32. Johnson JR, Bross P, Cohen M, et al: Approval summary: Imatinib mesylate capsules for treatment of adult patients with newly diagnosed philadelphia chromosome-positive chronic myelogenous leukemia in chronic phase. Clin Cancer Res 9: 1972-1979, 2003.

33. Cohen MH, Williams G, Johnson JR, et al: Approval summary for imatinib mesylate capsules in the treatment of chronic myelogenous leukemia. Clin Cancer Res 8: 935-942, 2002.

34. Joensuu H, Roberts PJ, Sarlomo-Rikala M, et al: Effect of the tyrosine kinase inhibitor STI571 in a patient with a metastatic gastrointestinal stromal tumor. N Engl J Med 344: 1052-1056, 2001.

35. Wen PY, Yung WK, Lamborn KR, et al: Phase I/II study of imatinib mesylate for recurrent malignant gliomas: North American brain tumor consortium study 99-08. Clin Cancer Res 12: 4899-4907, 2006.

36. Rubin BP and Duensing A: Mechanisms of resistance to small molecule kinase inhibition in the treatment of solid tumors. Lab Invest 86: 981-986, 2006.

37. Ma Y, Zeng S, Metcalfe DD, et al: The c-KIT mutation causing human mastocytosis is resistant to STI571 and other KIT kinase inhibitors; kinases with enzymatic site mutations show different inhibitor sensitivity profiles than wild-type kinases and those with regulatory-type mutations. Blood 99: 1741-1744, 2002. 